\title{
Clinical Efficacy of Conservative Treatment with Micronized Purified Flavonoid Fraction in Female Patients with Pelvic Congestion Syndrome
}

\author{
Rustem V. Akhmetzianov (D) · Roman A. Bredikhin
}

Received: June 25, 2021 / Accepted: August 18, 2021 / Published online: September 19, 2021

(C) The Author(s) 2021

\begin{abstract}
Introduction: Pelvic congestion syndrome (PCS) may be effectively managed with conservative treatment in certain patients. Treatment with venoactive drugs is common, but supportive data are limited. This study evaluated the clinical efficacy of micronized purified flavonoid fraction (MPFF) in women with PCS.

Methods: In a single-blind, placebo-controlled study, women with duplex ultrasound diagnosis of pelvic varicose veins (PVV) and PCS were randomized to MPFF $1000 \mathrm{mg}$ once daily or placebo for 2 months. Clinical manifestations of PCS were evaluated at baseline and end of treatment (M2) using three assessment tools: disease-specific quality of life (QoL) Pelvic Varicose Vein Questionnaire (PVVQ), Pelvic Venous Clinical Severity Score (PVCSS), and the Visual Analog Scale (VAS) for the main symptoms of the disease.
\end{abstract}

Supplementary Information The online version contains supplementary material available at https:// doi.org/10.1007/s40122-021-00312-6.

R. V. Akhmetzianov · R. A. Bredikhin Interregional Clinical Diagnostic Center, Department of Vascular Surgery, Kazan, Russia

R. V. Akhmetzianov $(\varangle) \cdot$ R. A. Bredikhin Kazan State Medical University, Department of Cardiovascular and Endovascular Surgery, Kazan, Russia

e-mail: arustemv@mail.ru
Results: A total of 83 women were included, 42 received MPFF and 41 received placebo.In the MPFF group, the mean global PVVQ QoL index decreased significantly from $45.1 \pm 14.7$ at baseline to $36.6 \pm 10.6$ at M2 (mean change: $8.2 \pm 10.4$ ); no significant change was observed in the control group (mean change: $-0.3 \pm 4.0)$. The between-group difference was statistically significant $(P<0.001)$. Compared with control, significant improvements were observed in all four QoL parameters (pain, physical, social, psychological, all $\mathrm{P}<0.001)$. The mean PVCSS summary score decreased significantly by $3.4 \pm 3.4$ in the MPFF group $(P<0.001)$ compared with a non-significant change of $-0.2 \pm 1.6$ in the control group (between-group difference $P<0.001$ ). In the MPFF group, improvements were statistically significant for 6 out of 10 clinical manifestations of PCS measured using the PVCSS, including pain (mean change from baseline: $0.5 \pm 0.7)$ heaviness $(0.4 \pm 0.7)$, discomfort $(0.6 \pm 0.7)$ and tenderness $(0.3 \pm 0.5)$. No significant improvements were observed in the control group. When measured by VAS, between-group differences were statistically significant for the overall summary score $(P<0.001)$ and for 8 out of 10 PCS symptoms, including: pain (mean MPFF change from baseline: $2.0 \pm 2.2)$, heaviness $(1.3 \pm 2.1)$, discomfort $(1.5 \pm 2.0)$, tenderness $(0.9 \pm 1.9)$, and edema $(1.3 \pm 2.1)$. 
Conclusion: In women with PCS, conservative treatment with MPFF was associated with improved QoL and reduced symptom severity. MPFF may be considered an effective and safe treatment option for PCS in routine clinical practice.

Keywords: Chronic pelvic pain; Chronic pelvic venous disorders; Daflon; Detralex; Micronized purified flavonoid fraction; Pelvic congestion syndrome; Pelvic varicose veins; Pelvic Varicose Veins Questionnaire; Quality of life; Venous Clinical Severity Score; Visual Analog Scale

\section{Key Summary Points}

Why carry out this study?

Pelvic congestion syndrome (PCS) as a result of pelvic varicose veins represents a form of chronic venous disease (CVD). However, while there is substantial evidence for the role of venoactive drugs in the management of CVD, data on their use in patients with PCS are limited.

This placebo-controlled study assessed the clinical efficacy of the venoactive agent micronized purified flavonoid fraction (MPFF) in female patients with PCS.

\section{What was learned from the study?}

Conservative treatment with MPFF was associated with improved quality of life and reduced symptom severity compared with control.

These results provide supporting data for the use of MPFF as a safe and effective treatment for PCS in routine clinical practice.

\section{INTRODUCTION}

Pelvic congestion syndrome (PCS) falls into the category of chronic pelvic venous disorders and represents a separate nosological entity, which is primarily diagnosed by vascular specialists and gynecologists. However, due to the very heterogeneous manifestations of the disease, patients are often diagnosed late, after multiple and varied consultations. Furthermore, despite recent advances in PCS research, the majority of specialists do not have a clear understanding of its clinical manifestations and treatment methods $[1,2]$.

PCS is widespread in the population. Its prevalence among women of reproductive age is about $6-15 \%$ [4], and its incidence shows no trend to decline. PCS is characterized by a dilation of the ovarian veins and intrapelvic venous plexuses [3], and can occur at any time in a woman's life, but is seen more frequently in multiparous women. Despite the number of comprehensive studies on this disorder, many issues relating to the diagnosis and treatment of PCS remain unresolved [5].

A variety of surgical, endovascular and conservative methods are available for the treatment of PCS [5]. As it represents a form of chronic venous disease (CVD), one of the main components of medical therapy is the prescription of venoactive drugs (VADs). Unfortunately, there is a lack of studies in the contemporary scientific literature, and most of those available include only limited numbers of patients and have focused on assessment of changes in the pain syndrome [6-10]. This simplified approach does not allow objective evaluation of PCS treatment: the manifestations of this disease are not limited only to chronic pelvic pain, but include a range of other symptoms. A further unresolved issue concerns patients with a socalled latent (from Latin "latentis"-hidden or invisible) form of the disease, who consider themselves healthy but have been diagnosed with anatomical and morphological signs of PCS in the absence of any complaints related to the condition.

The aim of the present study was to assess the clinical efficacy of the venoactive agent micronized purified flavonoid fraction (MPFF) in female patients with PCS. 


\section{METHODS}

\section{Study Design}

This prospective, randomized, single-blind, placebo-controlled study was carried out at the facilities of the Interregional Clinical Diagnostic Center, Kazan, Russia. The main inclusion criterion was the presence of pelvic varicose veins verified by transvaginal and transabdominal duplex ultrasound scanning (DUS) using the Voluson E8, Voluson E10 and LOGIQ E9 ultrasound systems (GE Healthcare, USA). PCS was diagnosed if the following were present: dilation of the ovarian vein by more than $5 \mathrm{~mm}$ associated with valvular insufficiency, and dilation of the pelvic plexus veins by more than $5 \mathrm{~mm}[11,12]$. Reflux was considered pathological if it persisted for more than $1 \mathrm{~s}$ and had a mean peak velocity greater than $5 \mathrm{~cm} / \mathrm{s}[11,12]$. Other inclusion criteria were age from 18 to 60 years, and written informed consent to participate in the study. Women were not included in the study if they had any of the following concomitant disorders: severe disease of the gastrointestinal tract or hematopoietic system; terminal stage cardiovascular, respiratory, renal or hepatic failure; grade IV malignancy; peripheral artery disease (PAD) of the lower extremities; any type of diabetes; or mental health disorder. In addition, the study did not include pregnant women at any gestational age, women who had given birth in the past 12 months, and breastfeeding mothers.

In order to exclude subjective factors, patients were eligible for inclusion only if they had not been previously examined by a vascular surgeon. Patients referred by a gynecologist with a preliminary diagnosis of PCS, either due to certain complaints or with asymptomatic forms revealed during screening, underwent examination in the Department of Ultrasound Diagnostics. Only after ultrasound verification of the disease were patients referred to a vascular surgeon.

At the general clinical examination, doctors recorded patients' complaints, medical history, presence, severity and nature of the pathological condition. Consecutive patients were then randomly allocated to receive MPFF at a dose of $1000 \mathrm{mg}$ once daily (study group) or placebo (control group). To ensure blinded treatment, the placebo glucose tablets were a similar color, size and shape to MPFF. All tablets were removed from their original blister packs and placed in opaque plastic bottles labeled"MPFF/placebo before dispensing to patients. Patients were advised to take their tablets with the morning meal. The duration of the continuous treatment course was 60 days.

\section{Patient Assessments and Outcome Measures}

Patients underwent a general clinical examination on days 0 and 61 of the study. At the screening visit, the results of ultrasound examination (performed within the 30 days prior to the screening visit) were evaluated.

Three quantitative methods were used to assess changes in severity of the main PCS symptoms over time. Quality-of-life (QoL) was assessed with the disease-specific QoL Pelvic Varicose Veins Questionnaire (PVVQ) [13], which is based on the well-known Chronic Venous Disease Quality of Life Questionnaire20 items (CIVIQ-20) [14]. The PVVQ covers the four main dimensions of QoL of patients with PCS: pain syndrome, physical, social and psychological well-being. Each of the 20 questions is scored using a 5-point scale to obtain a summary score ranging from 20 to 100: the higher the score, the lower the patient's QoL (Supplementary Table 1 ).

Assessment of treatment outcomes was standardized using the disease-specific Pelvic Venous Clinical Severity Score (PVCSS) for female patients with PCS (Supplementary Table 2) [15]. The prototype for this scale was the Venous Clinical Severity Score (VCSS) $[16,17]$. The PVCSS takes into account 10 major symptoms and signs of the disease, each of which is scored from 0 (no symptom) to 3 (maximum severity) for a maximum score of 30 , which indicates an extremely severe degree of disease.

Subjective manifestations of PCS symptoms were assessed using a Visual Analog Scale (VAS). 
The VAS is a self-reported scale consisting of a horizontal or vertical line, usually $10 \mathrm{~cm}$ long. An introductory question asks the patient to mark a point on the line that best refers to his or her pain. Patients were encouraged to assess the intensity of subjective manifestation of each symptom by scoring from 0 (no symptom) to 10 (the most extreme manifestation possible). The scores for each symptom were summed to obtain a summary score ranging from 0 to 100 .

\section{Statistical Analyses}

Statistical analysis of the data was carried out using the Microsoft Excel (Microsoft Corp., Redmond, WA, USA) and Statistica 10 (TIBCO Software Inc., Palo Alto, CA, USA; license No. AXAR212F599006FA-W) software packages. Within-group differences were compared using the Wilcoxon signed-rank test. To compare between-group differences in changes over time, the Mann-Whitney $U$ test was used. Differences were considered statistically significant when the $P$ value was $<0.05$. No multiplicity adjustments were made in view of the exploratory nature of the analyses.

\section{Ethical Considerations}

Ethics committee approval was obtained before the study from the local ethics committee of the Interregional Clinical Diagnostic Center (disposition No. 27 R-P 03/03/2014). All patients were informed prior to inclusion about the risks related to study drug intake and provided written informed consent.

All procedures were carried out in accordance with the ethical standards of the institutional and national research committee, as well as with the Declaration of Helsinki adopted in 1964, with its later amendments.

This trial was registered through the https:// clinicaltrials.gov site with the following identifier: NCT04512443.

\section{RESULTS}

The study enrolled 83 women with PCS of whom 42 were randomized to MPFF $1000 \mathrm{mg}$ once daily and 41 to matching placebo. Baseline clinical characteristics and demographic variables were mostly comparable for the two groups, with the exception of a greater proportion of women in the control group reporting use of contraceptives for up to 13 years (11 [26.9\%] vs 6 [14.3\%]) (Table 1).

Combined dilation of the ovarian veins and pelvic venous plexuses was present in 52 $(62.7 \%)$ women, and $31(37.3 \%)$ had an isolated dilation of the pelvic venous plexuses. No cases of isolated dilation of the ovarian veins were detected. Lesions were found to be unilateral in $18(22.6 \%)$ and bilateral in $65(77.4 \%)$ patients. Examples of ultrasound images of affected ovarian and left pampiniform plexus veins are shown in Figs. 1 and 2.

Patients presented with various complaints related to the manifestations of PCS and associated chronic pelvic venous disorders. Ten main clinical symptoms were identified: (1) pain, (2) feeling of heaviness, (3) lower abdominal discomfort, (4) pain in the sacrum and coccyx during prolonged sitting or standing, (5) dyspareunia, (6) menstrual disorders, (7) tenderness in the perineum, (8) edema in the perineum, (9) dysuria, (10) signs of atypical varicosis (Table 2). The symptoms were in agreement with those described in the PVCSS. After completing the questionnaires, all women reported the presence of at least two symptoms, even those who initially reported no complaints. The number of PCS symptoms ranged from 2 to 10, the mean number of symptoms was $6.9 \pm 2.6$.

\section{Change in Proportion of Women with PCS Symptoms}

The proportion of women with PCS symptoms in the control group remained unchanged or increased at 61 days and the mean number of symptoms per patient increased from $6.8 \pm 2.2$ at day 0 to $7.2 \pm 2.0$ at day 61 . In the MPFF group, the proportion of women with each 
Table 1 Baseline clinical characteristics and demographic data of the study participants

\begin{tabular}{|c|c|c|c|c|c|c|}
\hline \multirow[t]{2}{*}{ Parameter } & \multicolumn{2}{|c|}{ MPFF group $(n=42)$} & \multicolumn{2}{|c|}{ Control group $(n=41)$} & \multicolumn{2}{|c|}{ Total $(N=83)$} \\
\hline & Mean \pm SD & Range & Mean \pm SD & Range & Mean \pm SD & Range \\
\hline Age (years) & $34.7 \pm 4.9$ & $21-45$ & $35.8 \pm 5.9$ & $19-55$ & $34.4 \pm 5.0$ & $19-55$ \\
\hline Body mass index $\left(\mathrm{kg} / \mathrm{m}^{2}\right)$ & $22.3 \pm 2.6$ & $16.9-33.0$ & $23.2 \pm 3.1$ & $17.4-32.5$ & $22.7 \pm 2.9$ & $16.9-33.0$ \\
\hline Age of disease onset (years) & $29.3 \pm 5.2$ & $16-41$ & $30.0 \pm 6.5$ & $16-46$ & $29.8 \pm 5.9$ & $16-46$ \\
\hline Duration of disease (years) & $5.3 \pm 3.7$ & $0.5-20$ & $6.0 \pm 4.7$ & $0.5-23$ & $5.4 \pm 4.1$ & $0.5-23$ \\
\hline Risk factors & $1.7 \pm 0.9$ & $0-5$ & $1.5 \pm 0.8$ & $0-4$ & $1.6 \pm 0.9$ & $0-5$ \\
\hline Age at menarche (years) & $13.1 \pm 1.0$ & $9-17$ & $13.4 \pm 1.3$ & $11-18$ & $13.3 \pm 1.2$ & $9-18$ \\
\hline Duration of menstrual cycle (days) & $27.2 \pm 1.4$ & $24-31$ & $26.9 \pm 1.7$ & $21-30$ & $27.1 \pm 1.5$ & $21-31$ \\
\hline Duration of menstrual bleeding (days) & $4.9 \pm 1.2$ & $3-9$ & $5.7 \pm 1.2$ & $2-11$ & $5.2 \pm 1.2$ & $2-11$ \\
\hline Number of pregnancies & $2.5 \pm 1.2$ & $0-5$ & $2.9 \pm 1.3$ & $0-7$ & $2.6 \pm 1.3$ & $0-7$ \\
\hline Number of births & $1.7 \pm 0.8$ & $0-5$ & $1.7 \pm 0.8$ & $0-4$ & $1.7 \pm 0.8$ & $0-5$ \\
\hline Number of miscarriages & $0.1 \pm 0.2$ & $0-1$ & $0.3 \pm 0.5$ & $0-2$ & $0.2 \pm 0.3$ & $0-2$ \\
\hline Number of medical abortions & $0.6 \pm 0.8$ & $0-3$ & $1.0 \pm 1.0$ & $0-5$ & $0.8 \pm 0.9$ & $0-5$ \\
\hline
\end{tabular}

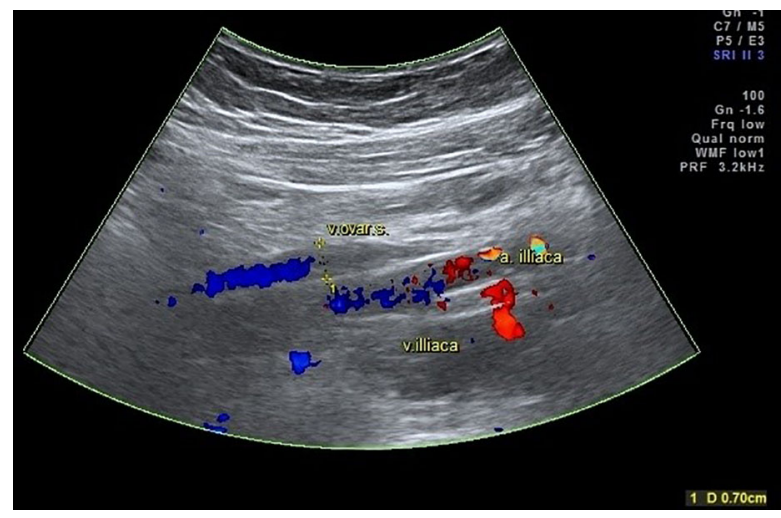

Fig. 1 Dilation of the left ovarian vein up to $0.7 \mathrm{~cm}$ in Color Doppler mode

clinical PCS manifestation was decreased after 60 days of treatment, with the exception of signs of atypical varicosis, which remained unchanged (Table 2). As a result, active treatment was associated with a reduction in the mean number of symptoms from $6.9 \pm 3.0$ at day 0 to $6.0 \pm 3.2$ at day $61, \mathrm{P}<0.05)$.

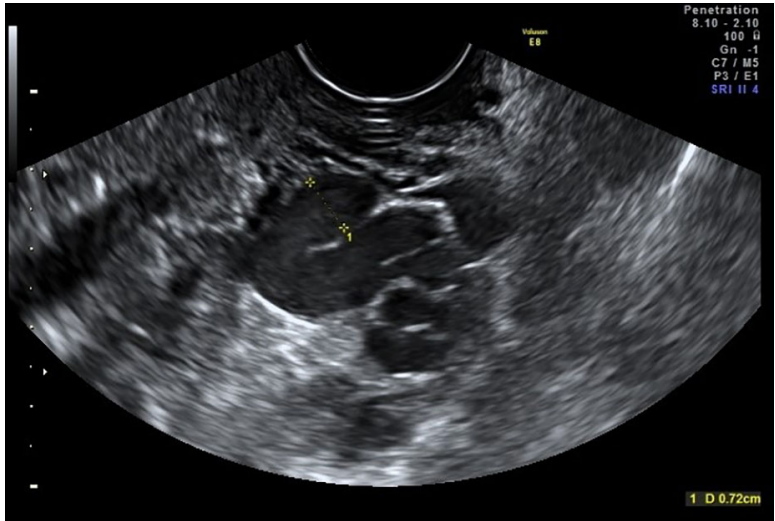

Fig. 2 Dilation of the veins of the left pampiniform plexus up to $0.72 \mathrm{~cm}$ in B-mode

\section{Changes in Quality-of-Life Parameters}

QoL parameters were assessed with the PVVQ; Table 3 illustrates PVVQ scores before and after treatment for the four QoL dimensions. In the control group, changes in the mean values of the physical, social and psychological parameters suggested a worsening QoL for patients in this group. The only positive change was a non- 
Table 2 Changes in the number (\%) of patients with symptoms during the study

\begin{tabular}{|c|c|c|c|c|c|}
\hline \multirow[t]{2}{*}{ Symptom } & \multirow{2}{*}{$\begin{array}{l}\text { Total }(N=83) \\
\text { Day } 0\end{array}$} & \multicolumn{2}{|c|}{ MPFF group $(n=42)$} & \multicolumn{2}{|c|}{ Control group $(n=41)$} \\
\hline & & Day 0 & Day 61 & Day 0 & Day 61 \\
\hline Lower abdominal pain & $73(88 \%)$ & $36(85.7 \%)$ & $31(73.8 \%)$ & $37(90.2 \%)$ & $37(90.2 \%)$ \\
\hline Lower abdominal heaviness & $69(83.1 \%)$ & $34(80.1 \%)$ & $29(69 \%)$ & $35(85.4 \%)$ & $38(92.7 \%)$ \\
\hline Pain in the sacrum and coccyx & $68(82 \%)$ & $34(80.1 \%)$ & $32(76.2 \%)$ & $34(82.9 \%)$ & $33(80.5 \%)$ \\
\hline Lower abdominal discomfort & $67(80.7 \%)$ & $32(76.2 \%)$ & $27(64.3 \%)$ & $35(85.4 \%)$ & $37(90.2 \%)$ \\
\hline Dyspareunia & $63(75.9 \%)$ & $29(69 \%)$ & $26(61.9 \%)$ & $34(82.9 \%)$ & $34(82.9 \%)$ \\
\hline Dysuria & $51(61.4 \%)$ & $33(78.6 \%)$ & $27(64.3 \%)$ & $18(43.9 \%)$ & $20(48.8 \%)$ \\
\hline Tenderness in the perineum & $49(59 \%)$ & $25(59.5 \%)$ & $18(42.9 \%)$ & $24(58.5 \%)$ & $26(63.4 \%)$ \\
\hline Edema in the perineum & $46(55.4 \%)$ & $25(59.5 \%)$ & $20(47.6 \%)$ & $21(51.2 \%)$ & $22(53.7 \%)$ \\
\hline Menstrual disorders & $45(54.2 \%)$ & $21(50 \%)$ & $20(47.6 \%)$ & $24(58.5 \%)$ & $27(65.9 \%)$ \\
\hline Signs of atypical varicosis & $38(45.8 \%)$ & $20(47.6 \%)$ & $20(47.6 \%)$ & $18(43.9 \%)$ & $20(48.8 \%)$ \\
\hline \multicolumn{6}{|c|}{ Mean number of symptoms per patient $($ mean $\pm S D)$} \\
\hline & $6.9 \pm 2.6$ & $6.9 \pm 3.0$ & $6.0 \pm 3.2$ & $6.8 \pm 2.2$ & $7.2 \pm 2.0$ \\
\hline
\end{tabular}

Table 3 Changes in the Pelvic Varicose Vein Questionnaire (PVVQ) quality-of-life parameters in the micronized purified flavonoid fraction (MPFF, $n=42)$ and control $(n=41)$ groups

\begin{tabular}{|c|c|c|c|c|c|}
\hline \multirow[t]{2}{*}{ Dimension } & \multicolumn{2}{|c|}{ PVVQ score (mean \pm SD) } & \multirow{2}{*}{$\begin{array}{l}\text { Change D61-D0 } \\
(\text { mean } \pm S D)\end{array}$} & \multirow{2}{*}{$\begin{array}{l}P \text { value } \\
\text { (intra-group) }\end{array}$} & \multirow{2}{*}{$\begin{array}{l}P \text { value } \\
\text { (inter-group) }\end{array}$} \\
\hline & Day 0 & Day 61 & & & \\
\hline \multicolumn{6}{|l|}{ MPFF group } \\
\hline Pain & $11.5 \pm 3.8$ & $9.3 \pm 2.8$ & $2.1 \pm 2.2$ & $<0.001$ & $<0.001$ \\
\hline Physical & $10.8 \pm 3.7$ & $8.7 \pm 3.0$ & $2.1 \pm 2.7$ & $<0.001$ & $<0.001$ \\
\hline Social & $10.1 \pm 5.0$ & $8.1 \pm 3.1$ & $2.1 \pm 3.5$ & $<0.001$ & $<0.001$ \\
\hline Psychological & $12.7 \pm 4.6$ & $10.6 \pm 4.0$ & $2.0 \pm 3.3$ & $<0.001$ & $<0.001$ \\
\hline Summary score & $45.1 \pm 14.7$ & $36.6 \pm 10.6$ & $8.2 \pm 10.4$ & $<0.001$ & $<0.001$ \\
\hline \multicolumn{6}{|l|}{ Control group } \\
\hline Pain & $13.0 \pm 4.6$ & $12.8 \pm 4.6$ & $0.1 \pm 1.2$ & NS & - \\
\hline Physical & $10.7 \pm 4.0$ & $10.8 \pm 3.5$ & $-0.2 \pm 1.3$ & NS & - \\
\hline Social & $9.2 \pm 4.0$ & $9.3 \pm 3.9$ & $-0.2 \pm 1.0$ & NS & - \\
\hline Psychological & $11.0 \pm 3.5$ & $11.0 \pm 3.4$ & $-0.1 \pm 1.8$ & NS & - \\
\hline Summary score & $43.8 \pm 13.0$ & $43.9 \pm 12.3$ & $-0.3 \pm 4.0$ & NS & - \\
\hline
\end{tabular}

SD standard deviation 
Table 4 Changes in the clinical manifestations assessed by the Pelvic Venous Clinical Severity Score (PVCSS) in the micronized purified flavonoid fraction (MPFF, $n=42)$ and control $(n=41)$ groups

\begin{tabular}{|c|c|c|c|c|c|}
\hline \multirow[t]{2}{*}{ Parameter } & \multicolumn{2}{|c|}{ PVCSS score (mean \pm SD) } & \multirow{2}{*}{$\begin{array}{l}\text { Change D61-D0 } \\
(\text { mean } \pm \text { SD })\end{array}$} & \multirow{2}{*}{$\begin{array}{l}P \text { value } \\
\text { (intra-group) }\end{array}$} & \multirow{2}{*}{$\begin{array}{l}P \text { value } \\
\text { (inter-group) }\end{array}$} \\
\hline & Day 0 & Day 61 & & & \\
\hline \multicolumn{6}{|l|}{ MPFF group } \\
\hline Pain & $1.4 \pm 0.9$ & $0.9 \pm 0.6$ & $0.5 \pm 0.7$ & $<0.001$ & 0.003 \\
\hline Heaviness & $1.2 \pm 0.9$ & $0.8 \pm 0.8$ & $0.4 \pm 0.7$ & 0.001 & 0.003 \\
\hline Discomfort & $1.3 \pm 1.0$ & $0.7 \pm 0.6$ & $0.6 \pm 0.7$ & $<0.001$ & $<0.001$ \\
\hline Pain in the sacrum and coccyx & $1.4 \pm 1.0$ & $1.0 \pm 0.9$ & $0.4 \pm 0.6$ & $<0.001$ & 0.012 \\
\hline Urination disorder & $1.2 \pm 1.0$ & $0.8 \pm 0.9$ & $0.4 \pm 0.8$ & 0.005 & 0.033 \\
\hline Atypical varicosis & $0.8 \pm 1.1$ & $0.6 \pm 0.9$ & $0.2 \pm 0.7$ & NS & NS \\
\hline Dyspareunia & $1.0 \pm 0.9$ & $0.8 \pm 0.8$ & $0.2 \pm 0.5$ & 0.008 & NS \\
\hline Menstrual disorders & $0.8 \pm 0.9$ & $0.7 \pm 0.9$ & $0.1 \pm 0.5$ & NS & NS \\
\hline Tenderness & $0.8 \pm 1.0$ & $0.4 \pm 0.7$ & $0.3 \pm 0.5$ & 0.001 & 0.024 \\
\hline Edema & $0.7 \pm 1.0$ & $0.4 \pm 0.7$ & $0.2 \pm 0.6$ & 0.018 & NS \\
\hline Summary score & $10.5 \pm 6.0$ & $7.1 \pm 4.6$ & $3.4 \pm 3.4$ & $<0.001$ & $<0.001$ \\
\hline \multicolumn{6}{|l|}{ Control group } \\
\hline Pain & $1.4 \pm 0.7$ & $1.4 \pm 0.8$ & $-0.0 \pm 0.4$ & NS & - \\
\hline Heaviness & $1.3 \pm 0.8$ & $1.4 \pm 0.9$ & $-0.1 \pm 0.4$ & NS & - \\
\hline Discomfort & $1.1 \pm 0.8$ & $1.2 \pm 0.8$ & $-0.2 \pm 0.5$ & NS & - \\
\hline Pain in the sacrum and coccyx & $1.3 \pm 1.0$ & $1.3 \pm 1.0$ & $0.0 \pm 0.4$ & NS & - \\
\hline Urination disorder & $0.8 \pm 1.0$ & $0.9 \pm 1.1$ & $-0.1 \pm 0.3$ & NS & - \\
\hline Atypical varicosis & $0.7 \pm 1.0$ & $0.7 \pm 0.9$ & $0.0 \pm 0.2$ & NS & - \\
\hline Dyspareunia & $1.3 \pm 1.0$ & $1.3 \pm 0.9$ & $-0.0 \pm 0.4$ & NS & - \\
\hline Menstrual disorders & $1.1 \pm 1.0$ & $1.1 \pm 1.0$ & $0.0 \pm 0.4$ & NS & - \\
\hline Tenderness & $1.0 \pm 1.0$ & $1.0 \pm 1.0$ & $0.0 \pm 0.4$ & NS & - \\
\hline Edema & $0.9 \pm 1.1$ & $0.8 \pm 1.0$ & $0.0 \pm 0.4$ & NS & - \\
\hline Summary score & $11.0 \pm 5.4$ & $11.2 \pm 5.6$ & $-0.2 \pm 1.6$ & NS & - \\
\hline
\end{tabular}

$S D$ standard deviation

significant improvement in the pain score by $0.1 \pm 0.8$. The global QoL index was improved in $12(29.3 \%)$ patients, unchanged in 13 (31.7\%), and worsened in $16(39 \%)$ patients. In contrast, MPFF treatment resulted in statistically significant improvements in all four QoL parameters at day 61: pain improved by
$2.1 \pm 1.6$, physical by $2.1 \pm 1.8$, social by $2.1 \pm 2.4$, and psychological by $2.0 \pm 2.5$ (all $P<0.05$ ) (Table 3). Between-group differences were statistically significant for all four QoL dimensions and for the overall summary score $(P<0.001)$. The global QoL index was improved in 38 (90.5\%) patients, unchanged in 
Table 5 Changes in pelvic congestion syndrome symptoms assessed by the visual analog scale (VAS) in the micronized purified flavonoid fraction (MPFF, $n=42)$ and control $(n=41)$ groups

\begin{tabular}{|c|c|c|c|c|c|}
\hline \multirow[t]{2}{*}{ Scale } & \multicolumn{2}{|c|}{ VAS score $($ mean $\pm S D)$} & \multirow{2}{*}{$\begin{array}{l}\text { Change } \\
\text { D61-D0 } \\
(\text { mean } \pm \mathrm{SD})\end{array}$} & \multirow{2}{*}{$\begin{array}{l}P \text { value (intra-group, } \\
\text { Wilcoxon signed-rank } \\
\text { test) }\end{array}$} & \multirow{2}{*}{$\begin{array}{l}P \text { value (inter-group, } \\
\text { Mann-Whitney } U \text { test) }\end{array}$} \\
\hline & Day 0 & Day 61 & & & \\
\hline \multicolumn{6}{|l|}{ MPFF group } \\
\hline Pain & $4.0 \pm 3.0$ & $2.0 \pm 2.0$ & $2.0 \pm 2.2$ & $<0.001$ & $<0.001$ \\
\hline Heaviness & $3.1 \pm 3.2$ & $1.8 \pm 2.3$ & $1.3 \pm 2.1$ & $<0.001$ & $<0.001$ \\
\hline Discomfort & $3.3 \pm 3.4$ & $1.8 \pm 2.4$ & $1.5 \pm 2.0$ & $<0.001$ & $<0.001$ \\
\hline $\begin{array}{l}\text { Pain in the } \\
\text { sacrum and } \\
\text { coccyx }\end{array}$ & $3.3 \pm 3.2$ & $1.9 \pm 2.3$ & $1.3 \pm 2.4$ & $<0.001$ & 0.013 \\
\hline $\begin{array}{l}\text { Urination } \\
\text { disorder }\end{array}$ & $2.4 \pm 2.9$ & $1.5 \pm 2.0$ & $1.1 \pm 2.4$ & $<0.001$ & $<0.001$ \\
\hline $\begin{array}{l}\text { Atypical } \\
\text { varicosis }\end{array}$ & $2.3 \pm 3.2$ & $1.6 \pm 2.5$ & $0.7 \pm 2.1$ & 0.007 & NS \\
\hline Dyspareunia & $3.1 \pm 3.2$ & $1.9 \pm 2.4$ & $1.3 \pm 2.1$ & $<0.001$ & $<0.001$ \\
\hline $\begin{array}{l}\text { Menstrual } \\
\text { disorders }\end{array}$ & $1.7 \pm 2.7$ & $1.5 \pm 2.6$ & $0.2 \pm 1.9$ & NS & NS \\
\hline Tenderness & $2.0 \pm 2.9$ & $1.1 \pm 1.9$ & $0.9 \pm 1.9$ & $<0.001$ & $<0.001$ \\
\hline Edema & $2.5 \pm 3.4$ & $1.2 \pm 1.9$ & $1.3 \pm 2.1$ & $<0.001$ & $<0.001$ \\
\hline Summary score & $27.6 \pm 23.4$ & $16.3 \pm 18.0$ & $11.6 \pm 14.8$ & $<0.001$ & $<0.001$ \\
\hline \multicolumn{6}{|l|}{ Control group } \\
\hline Pain & $4.8 \pm 3.3$ & $4.8 \pm 3.2$ & $0.0 \pm 1.1$ & NS & - \\
\hline Heaviness & $4.2 \pm 2.9$ & $4.4 \pm 3.1$ & $-0.3 \pm 1.0$ & NS & - \\
\hline Discomfort & $3.7 \pm 2.8$ & $4.1 \pm 3.0$ & $-0.4 \pm 1.0$ & 0.008 & - \\
\hline $\begin{array}{l}\text { Pain in the } \\
\text { sacrum and } \\
\text { coccyx }\end{array}$ & $4.2 \pm 3.4$ & $4.0 \pm 3.1$ & $0.2 \pm 1.0$ & NS & - \\
\hline $\begin{array}{l}\text { Urination } \\
\text { disorder }\end{array}$ & $2.3 \pm 3.2$ & $2.3 \pm 3.2$ & $-0.1 \pm 1.0$ & NS & - \\
\hline $\begin{array}{l}\text { Atypical } \\
\text { varicosis }\end{array}$ & $2.2 \pm 3.1$ & $2.0 \pm 2.9$ & $0.49 \pm 1.2$ & NS & - \\
\hline Dyspareunia & $4.3 \pm 3.7$ & $4.4 \pm 3.5$ & $-0.1 \pm 1.2$ & NS & - \\
\hline $\begin{array}{l}\text { Menstrual } \\
\text { disorders }\end{array}$ & $2.8 \pm 3.4$ & $3.0 \pm 3.4$ & $-0.1 \pm 1.1$ & NS & - \\
\hline Tenderness & $2.4 \pm 3.1$ & $2.9 \pm 3.3$ & $-0.5 \pm 1.3$ & 0.019 & - \\
\hline Edema & $2.2 \pm 3.1$ & $2.5 \pm 3.1$ & $-0.3 \pm 1.1$ & NS & - \\
\hline
\end{tabular}


Table 5 continued

\begin{tabular}{|c|c|c|c|c|c|}
\hline \multirow[t]{2}{*}{ Scale } & \multicolumn{2}{|c|}{ VAS score $($ mean $\pm S D)$} & \multirow{2}{*}{$\begin{array}{l}\text { Change } \\
\text { D61-D0 } \\
(\text { mean } \pm S D)\end{array}$} & \multirow{2}{*}{$\begin{array}{l}P \text { value (intra-group, } \\
\text { Wilcoxon signed-rank } \\
\text { test) }\end{array}$} & \multirow{2}{*}{$\begin{array}{l}P \text { value (inter-group, } \\
\text { Mann-Whitney } U \text { test) }\end{array}$} \\
\hline & Day 0 & Day 61 & & & \\
\hline Summary score & $33.1 \pm 22.2$ & $34.5 \pm 23.6$ & $-1.0 \pm 5.2$ & NS & - \\
\hline
\end{tabular}

$3(7.1 \%)$, and worsened in $1(3.3 \%)$ patient. The mean value of the global QoL index was significantly improved by $8.2 \pm 7.0$ compared with baseline $(P<0.05)$.

\section{Changes in Quantitative Assessment of PCS Symptoms}

Quantitative changes in the severity of the clinical manifestations of PCS assessed using the PVCSS are shown in Table 4. In the control group, there were no statistically significant improvements in symptom severity and the overall summary score remained essentially unchanged $(11.0 \pm 4.7$ at day 0 and $11.2 \pm 4.8$ at day 61 , a difference of $-0.2 \pm 1.1$ ). The negative mean difference in the summary indexes before and after treatment suggested an absence of positive changes in the PCS course in controltreated patients. In contrast, MPFF was associated with statistically significant improvements in 6 of the 10 symptoms at day 61 and an improvement in the overall summary score from $10.5 \pm 5.0$ at day 0 to $7.1 \pm 4.0$ at day 61 , a difference of $3.4 \pm 2.5(P<0.05)$; betweengroup difference $(P<0.001)$.

\section{Changes in Subjective Assessment of PCS Symptoms}

Subjective assessments of the intensity of PCS symptoms before and after treatment according to the VAS are presented in Table 5. In the control group, mean symptom scores essentially remained the same or worsened over the course of the study, with no statistically significant change from day 0 . There was a slight increase in the mean summary score of VAS from $35.6 \pm 19.6$ at day 0 to $35.9 \pm 20.8$ at day 61 , which indicated an absence of positive changes in the PCS course in these patients. At the individual level, the clinical status was improved in 18 (43.9\%), unchanged in 1 (2.4\%), and worsened in $22(53.7 \%)$ patients.

In the group treated with MPFF, mean scores for all symptoms improved significantly from day 0 to day 61 , with the exception of atypical varicosis and menstrual disorders which showed a trend towards improvement (Table 5). Between-group differences were statistically significant for 8 out of the 10 symptoms and for the overall summary score $(P<0.001)$. The mean VAS summary score almost halved from its baseline value from $30.1 \pm 20.1$ at day 0 to $17.8 \pm 14.4$ at day 61 , a difference of $12.7 \pm 10.9 \quad(P<0.05)$. Clinical status was improved in 39 (92.9\%), unchanged in 2 (4.7\%), and worsened in 1 (2.4\%) patient.

\section{Safety and Tolerability}

No complications or adverse events were observed during the study. One patient in the MPFF group reported dyspeptic symptoms in the form of discomfort in the epigastric region and increased bloating during the first 2 days of taking the drug. These symptoms spontaneously resolved by the third day of treatment.

\section{DISCUSSION}

This study provides important information on the spectrum of symptoms observed in patients with PCS and their amelioration with pharmacotherapy. None of the 83 women in this study, including those who considered themselves to be completely healthy, were free from the manifestations of PCS. All women were actively 
identified as having at least two PCS symptoms at baseline. However, in order for the patients to recognize some of the disease symptoms, they needed to complete all the items of the proposed questionnaires. When treating patients with a lack of active complaints, the disease may therefore be more accurately described as a painless form of PCS rather than a latent form of the disease.

The presence of PCS symptoms, even if not painful, can have an important impact on patient QoL and provides the rationale for prescribing conservative therapy as a first step in all women diagnosed with PCS, particularly as the majority will still be of child-bearing age (mean age in the current study was 34 years). Medical treatment of PCS includes psychotherapy, analgesics, nonsteroidal anti-inflammatory drugs, the vasoconstrictive agent dihydroergotamine, medical suppression of ovarian function with progestins (contraceptives, hormone replacement therapy, danazol) and gonadotropin-releasing hormone (GnRH) agonists, and venoactive drugs [5]. Treatment is aimed at symptomatic pain relief, elimination of the microcirculatory and hemorheological disorders, inhibition of inflammation, increased venous tone and improved lymphatic drainage [18].

PCS is an important cause of chronic pelvic pain in women and is responsible for $2-10 \%$ of gynecological visits [19]. One of the main pathophysiological mechanisms underlying the development of pelvic pain in women with PCS is disturbance of venous hemodynamics due to blood stasis in the pelvis. Venoactive drugs containing the bioflavonoids diosmin and hesperidin, play a key role in the medical therapy of PCS. Previous studies have shown that by improving venous tone, MPFF may restore pelvic circulation and provide relief from the symptoms of chronic pelvic pain and a feeling of heaviness in the pelvic area [6-9].

Findings from the current placebo-controlled study provide evidence for a positive effect of therapy on the clinical course of PCS after 2 months of monotherapy with MPFF. Compared with the control group, patients treated with MPFF demonstrated statistically significant decreases at 2 months in both the number and severity of most PCS symptoms, with associated improvements in QoL, regardless of the assessment method used.

Treatment benefits included a reduction in the pain syndrome, improvements in physical and social activity parameters, and normalization of the psychological parameter, which together improve QoL. The absence of positive outcomes in the control group suggests that patients were dissatisfied with the results achieved and had unfulfilled treatment expectations, which could explain their worse ranking of health status at the time of treatment completion compared with baseline.

Although limited by its small sample size and single-blind design, this randomized, placebocontrolled study provides valuable information on the efficacy and safety of MPFF in real-life practice in a cohort of women with PCS who were well-matched at baseline in terms of clinical and demographic characteristics. Unlike most previous studies, the current analysis was not limited to assessment of painful complaints, but evaluated the efficacy of treatment on the full range of PCS symptoms and included women with anatomical and morphological signs of PCS in the absence of pain symptoms. The study therefore adds to the limited existing body of literature on the management of PCS symptoms in women.

\section{CONCLUSION}

The management of women with newly-diagnosed PCS should start with the use of conservative treatments followed by an assessment of their efficacy, before more invasive options are considered. The routine administration of MPFF at a daily dose of $1000 \mathrm{mg}$ for 2 months is an effective and safe approach to the conservative management of PCS and may be considered a key component of basic pharmacotherapy in all female patients with this condition.

\section{ACKNOWLEDGEMENTS}

The authors thank all the patients for their participation in this study. 
Funding. The study and the journal's Rapid Service Fee were funded by Servier, France.

Editorial Assistance. Editorial assistance was provided by Jenny Grice, BSc and funded by Servier, France.

Authorship. All named authors meet the International Committee of Medical Journal Editors (ICMJE) criteria for authorship for this article, take responsibility for the integrity of the work as a whole, and have given their approval for this version to be published.

Author Contributions. Study concept - R.V. Akhmetzianov, R.A. Bredikhin. Patient enrollment - R.V. Akhmetzianov, R.A. Bredikhin. Writing manuscript - R.V. Akhmetzianov. Statistical analysis - R.V. Akhmetzianov.

Disclosures. RV Akhmetzianov and RA Bredikhin have nothing to disclose.

Compliance with Ethics Guidelines. The ethics committee approval was obtained before the study from the local ethics committee of the Interregional Clinical Diagnostic Center (disposition No. 27 R-P 03/03/2014). All patients were informed prior to inclusion about the risks related to study drug intake and provided written informed consent. All procedures were carried out in accordance with the ethical standards of the institutional and national research committee, as well as with the Declaration of Helsinki adopted in 1964, with its later amendments. This trial was registered through the https://clinicaltrials.gov site with the following identifier: NCT04512443.

Data Availability. The datasets generated during and/or analyzed during the current study are available from the corresponding author on reasonable request.

Open Access. This article is licensed under a Creative Commons Attribution-NonCommercial 4.0 International License, which permits any non-commercial use, sharing, adaptation, distribution and reproduction in any medium or format, as long as you give appropriate credit to the original author(s) and the source, provide a link to the Creative Commons licence, and indicate if changes were made. The images or other third party material in this article are included in the article's Creative Commons licence, unless indicated otherwise in a credit line to the material. If material is not included in the article's Creative Commons licence and your intended use is not permitted by statutory regulation or exceeds the permitted use, you will need to obtain permission directly from the copyright holder. To view a copy of this licence, visit http://creativecommons.org/licenses/by$\mathrm{nc} / 4.0 /$.

\section{REFERENCES}

1. Borghi C, Dell'Atti L. Pelvic congestion syndrome: the current state of the literature. Arch Gynecol Obstet. 2016;293(2):291-301.

2. Asciutto G. Pelvic vein incompetence: a review of diagnosis and treatment. Phlebolymphology. 2012;19(2):84-90.

3. Stoyko-Yu M, Kirienko AI, Zatevakhin II, et al. Diagnostics and treatment of chronic venous disease: guidelines of Russian Phlebological Association. Flebologiya. 2018;3:146-240. https://doi.org/ 10.17116/flebo20187031146.

4. Howard FM. Chronic pelvic pain. Obstet Gynecol. 2003;101:594-611.

5. Antignani PL, Lazarashvili Z. Monedero JL, et al. Diagnosis and treatment of pelvic congestion syndrome: UIP consensus document. Int Angiol. 2019; 38(4):265-83.

6. Taskin O, Uryan I, Buhur A, et al. The effects of Daflon on pelvic pain in women with Taylor Syndrome. J Am Assoc Gynecol Laparosc. 1996;3:S49.

7. Simsek M, Burac F, Taskin O. Effects of micronized purified flavonoid fraction (Daflon) on pelvic pain in women with laparoscopically diagnosed pelvic congestion syndrome: a randomized trial. Clin Exp Obstet Gynecol. 2007;34(2):96-8.

8. Burac F, Guduz T, Simsek M, Taskin O. Chronic pelvic pain associated with pelvic congestion syndrome and the benefit of Daflon 500 mg: a review. Phlebolymphology. 2009;16(3):290-4. 
9. Gavrilov SG, Moskalenko YP, Karalkin AV. Effectiveness and safety of micronized purified flavonoid fraction for the treatment of concomitant varicose veins of the pelvis and lower extremities. Curr Med Res Opin. 2019;35(6):1019-26.

10. Taskin O, Sahin L, Gavrilov S, Lazarashvili Z. Medical treatment of pelvic congestion syndrome. Phlebolymphology. 2016;23:146-53.

11. Champaneria R, Shah L, Moss J, et al. The relationship between pelvic vein incompetence and chronic pelvic pain in women: systematic reviews of diagnosis and treatment effectiveness. Health Technol Assess. 2016;20(5):1-108.

12. Sharma K, Bora MK, Varghes J, et al. Role of trans vaginal ultrasound and Doppler in diagnosis of pelvic congestion syndrome. J Clin Diagn Res. 2014;8(7):OD05-7.

13. Akhmetzianov RV, Bredikhin RA, Fomina EE. Quality of life assessment in patients with pelvic varicose veins. Flebologiya. 2019;13(2):133-9 (In Russ).

14. Launois R, Reboul-Marty J, Henry B. Construction and validation of a quality of life questionnaire in
Chronic Lower Limb Venous Insufficiency (CIVIQ) Quality. Life Res. 1996;5(6):539-54.

15. Akhmetzyanov RV, Bredikhin RA, Fomina EE, Ignatyev IM. Method of determining disease severity in women with pelvic varicose veins. Angiol Sosud Khir. 2019;25(3):79-87 (In Russ).

16. Vasquez MA, Munschauer CE. Venous Clinical Severity Score and quality-of-life assessment tools: application to vein practice. Phlebology. 2008;23(6):259-75.

17. Kakkos SK, Rivera MA, Matsagas MI, et al. Validation of the new venous severity scoring system in varicose vein surgery. J Vasc Surg. 2003;38(2): 224-8.

18. Bush R, Comerota A, Meissner M, Raffetto JD, Hahn SR, Freeman K. Rec-ommendations for the medical management of chronic venous disease: the role of Micronized Purified Flavanoid Fraction (MPFF). Phlebology. 2017;32(1 suppl):3-19.

19. Cheong YC, Smotra G, Williams ACDC. Non-surgical interventions for the management of chronic pelvic pain. Cochrane Database of Syst Rev. 2014;3: CD008797. 